\title{
ONOMASTICA TERGESTINA NEL TRECENTO
}

\begin{abstract}
Buona parte dei cimelj si deve però sempre agli estri popolari, in quanto rinviene a soprannomi e a invettive.
\end{abstract}

G.I. Ascoli

Dallo spoglio dei nomi personali attestati nei sette codici di entrate e uscite conservati presso l'Archivio Capitolare di S. Giusto in Trieste e che riguardano uno dei secoli più ricchi di testimonianze storiche della città giuliana (1307-1406), risultano numerose forme cognominali e soprannominali espresse nel registro linguistico tergestino. La mancata pubblicazione del dizionario linguistico tergestino di Mario Doria ${ }^{1}$ rende impossibile un raffronto con il materiale lessicale tergestino fino a oggi noto, per cui si basa, a fini comparativi, esclusivamente sui due repertori lessicali tergestini dal Doria finora publicati (Elementi friulaneggianti nel dialetto triestino, in Italia linguistica nuova ed antica II, Galatina 1978, 329-405; Nuovi materiali per lo studio degli elementi lessicali friulaneggianti del dialetto triestino, in Archivio per l'Alto Adige LXVII, 1979 (Studi in memoria di Carlo Battisti editi dall'Istituto di Studi per l'Alto Adige), Firenze 1979, 65-100); sul Dizionario del dialetto muglisano di Diomiro Zudini e Pierpaolo Dorsi (Casamassima, Udine 1981) e sui due grandi vocabolari friulani: Il nuovo Pirona di Giulio Andrea Pirona, Ercole Carletti e Giovanni Battista Corgnali (Udine 1935, rist. 1977) e il Vocabolario della lingua friulana di Giorgio Faggin (Del Bianco, Udine 1985).

Impossibile risulta perciò valutare l'apporto quantitativo e qualitativo delle forme onomastiche qui di seguito elencate per una più approfondita conoscenza del tesoro lessicale tergestino; ce ne dogliamo, ma desideriamo allo stesso tempo sottolineare che la nostra incompetenza in materia ci consente di offrire soltanto un repertorio di nomi personali con i riferimenti alle opere lessicografiche citate.

Elencheremo pertanto le forme nominali - siano esse cognominali o soprannominali - in ordine alfabetico corredandole esclusivamente con i dati riguardanti le fonti e i raffronti con le opere citate, sperando che anche ciò possa costituire un modesto apporto alla conoscenza della civiltà linguistica e storica tergestina, che Trieste

1 Mario Doria, Lessico - concordanza del dialetlo tergestino moderno, cfr. M. Doria, Da Carlomagno a Maria Teresa. Saggio di un lessico delle origini della neolatinità triestina, in Archeografo Triestino S. IV, vol. XLIX (XCVII della Raccolta), 1989, 259-274 (lettera A). 
tende di norma a trascurare, mentre fervono studi e iniziative attinenti al triestino, parlata locale derivata dal veneziano coloniale e quindi seriore e secondaria rispetto al tergestino.

\section{Repertorio}

Bech. Martinus Bech (R. 1310, c. 5v.); soprannome corrispondente allo zoonimo tergestino *bek, mugl. bek. Si veda pure Sonça de bech.

Berlofa. Gen. Benevenute Berlofe (C 1368, c. 58r.); seguendo le tracce offerteci da DORIA 1987, 67 s.v. *berloca e 560 s.v. șberlefo, alle quali si rimanda, *berlofa potrebbe essere un appellativo tergestino corrispondente, per significato, a 'sberleffo'; cfr. FAGGIN 73 berlade (anche sberlade) 'grido, urlo, strillo', berlete 'strombazzamento, vanteria' e altri vocaboli ancora, tutti derivati da FAGGIN 72 berlâ (anche sberlâ) 'gridare, strillare, vociare, urlare'.

de la Bestia, de lis Bestiis, a Bestiis. Martinus de (la) Bestia/de (lis) Bestiis/a Bestiis (R 1308-1310 e Q [1315]-1320), Marticha de la Bestia (Q 1316-1320); altre attestazioni: PARENTIN 1321 Ivano a Bestijs; Gregorio a Bestijs suo figlio; SSMM 1344, 673, c. 4r Dominica Abestiis. Un Marinus de lis bestiis è citato da G.I. Ascoli, Il dialetto tergestino, in Archeografo Triestino, 1889, 245-263, a pag. 251 quale esempio di plurale sigmatico.

Il soprannome, attestato nell'arco di 36 anni, cioè di due generazioni, coinvolge tutto un gruppo familiare, ma ciononostante sembra non assumere la valenza di cognome. La famiglia è indubbiamente slovena (Martiha e Ivan sono forme nominali slovene, Martinus e Dominica sono agionimi comuni a individui di ogni derivazione etnica presenti in Trieste; Marticha è presumibilmente figlio di martinus e la differente forma nominale evita l'identica denominazione di padre e figlio, battezzati con lo stesso agionimo e ambedue censuari del Capitolo); il soprannome è stato loro evidentemente conferito dalla popolazione romanza della città. Per l'uso prevalente del soprannome con forme introdotte dalle preposizioni de e $a$ e dall'articolo la/lis sembra di potersi escludere ogni valenza metaforica ("Bestia, dicesi per agg. a uomo bestiale, che fa azione da bestia“, BOERIO 77) per rimanere nell'ambito della primaria valenza semantica dell'appellativo, cioè di persone che avevano a che fare con/che allevavano animali. Va inoltre rilevato che le forme del plurale sigmatico consentono di individuare nel soprannome una forma tergestina.

Blanc. Ben(e)venut(t)us/Benvinuto (quondam) presbiteri Blanc(h)i/Blanco/Blanc (R 1309-1310 e Q 1316); Bridonus Blanchi (C 1390, c. 7r), attestato già in BM 1354 Bridonus Albus; Marinus quondam fily Blanchi (C 1406, c. 39v.); ulteriori attestazioni: SSMM 1304, 673, c. 34r., 1308, 673, c. 23r. segg., 1311, 673, c. 16r. segg., 1313,673 , c. 13r. segg. (domino) Mathia Bla(n)co (de Tergesto); 1308, 673, c. 24v., 1311,673 , c. $18 \mathrm{r}$. segg. 1324, 666, 1344, 674, c. 1r. segg. (dominus) 
Antonius/Toncius/Tençe/Tontius (filius domini) Mathie Blanchi/Albi; 1315, 673, c. 36r. Marcius filius Dominici de Blanco.

Originariamente soprannome, poi nome diffusamente cognominizzato, corrispondente all'aggettivo tergestino *blank 'bianco', cfr. mugl. blank, bläink, FAGGIN 82 blanc; Albus è, per calco, l'equivalente forma dotta latina.

Brumba. Maurus Brumba (C 1360, c. 18v.); un appellativo tergestino *brumba, o più correttamente *brunba, troverebbe riscontro nel sostantivo pl. muglisano brónbui 'borborigmi, gorgoglii intestinali', nel verbo friulano brumbulâ 'rumoreggiare, brontolare' (FAGGIN 116), ma anche in un più lato bròmbola, bròmbolo, brombolar, attestato con molti significati nel veneziano (BOERIO 101, 102), triestino e istro-veneto (DORIA 1987, 94, 95).

Chauxe. Iacobus dictus Chauxe (C 1369, c. 61r.); unico riferimento possibile sembra essere la contrata (de) Chiause nel LIBRO DI PERTICATIONI del notarius I $u$ stus Raviza del 1525 (Archivio Diplomatico, Trieste, segn. B A 19) assieme alla contrata (de) Chiausich, oggi Velike C̆avse e Male C̆avse sulla fascia costiera a ponente di S. Croce, zona ricca di vigneti (Tržaško ozemlje, Ljubljana-Trst 1978, C3). Se ne può ipotizzare la derivazione dall'appellativo tergestino čaf 'testa, capo' (DORIA, Elementi 361), ma anche 'tralcio, sarmento', cfr. FAGGIN 295-297 s.v. čhâv, pl. čhâvs, e mugl. $21 \check{c} a f$, con semivocalizzazione della $v(f)$ finale nella composizione prima di una consonante (cfr. mugl. 26 caudístra). All'appellativo tergestino si sono seriormente aggiunte le formanti slovene - $e$ (acc. pl. nella locuzione grem $u$ Ćáuse) o $-i c$. La $x$ della forma soprannominale indica la palatalizzazione della s: Čáuše, spiegabile facilmente in più modi: sostituzione della $s$ romanza, analogia o altre ancora.

Cisilin. per mortem nepote Cisilini (C 1360, c. 18r.); corrisponde a un appellativo tergestino *čisilin, cfr. FAGGIN 173 cisilin 'rondinino, rondinotto'.

Fresora. ser Mathia Fresora (C 1404, c. 22v.); corrisponde a un appellativo tergestino *fresòra 'padella', cfr. mugl. 48 frisóra, *fresóra.

Gras(s)is C(h)astegnis, G(h)astegnis. a Gras(s)is C(h)astegnis (R [1310], cc. 11r. e 35r., a Grasis Casteneis/Castegnis (Q 1318-1319), presumibilmente identico a Iacogna Grasses Castagnes (R 1310, c. 50r. e Q 1316-1319; Lucia Grasse Castane/de Grassis G(h)astegnis (RP 1350, D 1357 e C 1360); il soprannome 'castagne grasse, grosse' è espresso in tergestino con il plurale sigmatico.

Il sostantivo dimostra tre aspetti: *casteña, *castaña, *gasteña; unici riferimenti lessicali sono mugl. 70 *kastańol e FAGGIN 149 castagn 'castano, marrone rossiccio', castagnolâr 'ippocastano', castagnole 'frutto dell'ippocastano'. Ma l'oscillazione grafica $\mathrm{Ca} / \mathrm{Ga}$ : Cha/Gha non ci esime dal tenere in considerazione pure i lessemi mugl. 26 častenár 'castagno', častína 'castagna'.

Ioven. Presbiter Nicolaus de Ioveno (C 1356, c. 4r.); Odorlicus/Voldericus (de) Ioven (C 1368, C 1369 e C 1404); *ioven è aggettivo tergestino 'giovane', cfr. FAGGIN 562 ğòvin. 
Masclo. Dominicus Masclo (R 1308 e Q 1318-1320); ser Amiço Masclo (PR 1348 e C 1368); altre attestazioni: 1327 Albericus Masculus not.malef. (MAGISTRATU$\mathrm{RE}$ ); corrisponde al sostantivo tergestino *másklo, cfr. mugl. 95 másklo 'maschio d'animale'.

de lis Molis/Muelis/Moli. Pasca de Molis/Moliis (R 1308-1310 e Q 1315); Simon de lis Molis/de Limolis/de Limoli (D 1357); Ciprianus/Civrianus de Limol(l)is/de lis Muelis (C 1366-1370 e M 1383); Dominicus de Limoli (C 1369); Francisca delis Molis/de Lismolis (C 1389-1390); altre attestazioni: Dominica Coya de Lismolis (BM 1354).

Plurale sigmatico dell'appellativo tergestino *muela o *muola, cfr. mugl. 102 muóla 'macina', FAGGIN 838 muèle 'macina, mola', corrispondente al veneziano mola 'mola, macina' (BOERIO 420), originario soprannome metonimico per un artigiano che produceva mole o per un mugnaio.

Moç. Almericus (dictus) Moç(o) (R [1308] - 1310 e Q 1320). Soprannome corrispondente al nome di mestiere tergestino *moz 'mozzo', cfr. FAGGIN 835 moz 'mozzo', in concorrenza con la forma italiana mozzo.

Muiul(l)o. Andreas Muiul(l)o (R 1309-1310); corrisponderebbe a un appellativo tergestino *mujùl, cfr. FAGGIN 839 mujûl 'mozzo della ruota'; soprannome metonimico.

Nainis. Almericus Nainis/Nagnis/Naynis (R [1309]-1310 e Q 1319); soprannome o cognome con forma del plurale sigmatico dell'appellativo tergestino *náina ? , cfr. mugl. 104 náina 'nenia' e DORIA 1987, 396 *nàina 'nenia, piagnisteo'; al Doria si rimanda per la diffusione e l'etimologia.

Niblo, de Niblis. Stefanus/Stephanus Niblo (R $1308-1310$ e Q 1320); Ben(e)venut(t)us Niblo (R 1309-1310 e Q 1316-1320); Çanetullus Niblo (R 1309); Franciscus Niblo (R 1309 e Q 1316-1320); Iohannes Niblo (M 1348); Andreas Nibli (C 1359); Simon Nibli (C 1359-1360); Iacobus Nibli (C 1360); presbiter Iacobus de Niblis (M 1383, C 1390-1399 e C 1404-1406); Mateus de Niblis (M, data non accertabile); ulteriori attestazioni: Dnicus. Niblo, Baro Niblo, Jo.Niblo (1202 PATRIZI); e frequenti altri in PARENTIN, MAGISTRATURE, HORTIS, BLOISE, PILLON, SSMM e MARSICH (1314-1436).

Il nesso $b l$ induce a considerare il cognome di impronta tergestina; gli unici raffronti possibili sono lo zoonimo nibli 'nibbio' (FAGGIN 864) o, più probabilmente, l'antroponimo Nibil 'Annibale' (ibid.).

Pangel, Pangil. per mortem uxoris Pangeli (C 1360, c. 21v.); Pascholus Pangili (C 1366, c. 45v.); il soprannome corrisponderebbe a un appellativo tergestino *pangel, cfr. FAGGIN $904 I^{\circ}$ pangel [-gèl] '1. pennecchio, roccata; 2. panno di lana, pannolano; 3. pannolino; 4. pennacchio, striscia...' e ibidem $2^{\circ}$ pangel [-gèl] ' 1 . panetto di burro; 2. tenero come il burro,...; 3. una pasta d'uomo...'. 
Paveia, Pavegla. Vitale Paveya/Paveia/Pavjan[o] (R 1309-1310 e Q 1316-1320); Andreas Pavegle/Paveie (C 1368); ulteriori attestazioni: notaio Mauro Paneija (1324 PARENTIN), Florio Paneya (1343 PARENTIN); marinus paueya notarius (1343 HORTIS); forse Vi. Pavilla (1202 PATRIZI).

Coincide con un sostantivo tergestino 'paveia 'farfalla', cfr. mugl. 116 pavéğa 'tignola del grano', pavee 'farfalla' (FAGGIN 937); la scrittura Pavegla corrisponde al veneziano pavegia 'farfalla' (BOERIO 483).

Paver. Dominicus Paver (M 1350, c. 13r.); corrisponde a un sostantivo tergestino *paver e mugl. 116 pavér 'lucignolo', cfr. FAGGIN 937 pavêr'1. stoppino, lucignolo; 2. moccio, mòccolo; 3. persona magrissima; 4. giunco da stuoie, giunco da palude'.

Pesant. de morte uxoris Pesa[nt] (Q 1318, c. 34v.); domina Nicholota de Pesant(o) (D 1357). La forma fa supporre un aggettivo tergestino *pesànt, cfr. FAGGIN 953 pesant '(anche fig.) pesante': per spiegare un originario soprannome Pesant si veda BOERIO 495 pesante 'pesante'; Omo pesante 'noioso; fastidioso; ricadioso; molesto' e la lettera dell'arcidiacono di Kamnik mons. Ferri al Patriarca Arcivescovo di Udine in data 27 febbraio 1686 (citata in P. Merkù, Zasebna slovenščina v 17. stoletju, in Slavistična revija, Ljubljana 1982, 121-150) dove pesante significa 'importante': “... conosciutolo dalla Cancelleria d'Ongheria numoso e pesante... “.

Piulo, Piuli. Iohannes Piulo/Piuli (R 1310, cc. 4r., 12v. e 37v.); altra attestazione: B. Piùla (1202 PATRIZI). Con riserva si accenna a mugl. 124, 130 pojóul, puzóul 'parapetto, appoggiatoio', e FAGIN 988, 1050 piûl, pujûl 'ballatoio, terrazzino, balcone'.

Piçol, Piçul. a Cotiano Piçol, a Canciano Pravo (Q [1315]-1320); Dominicus Todesco Piçul (C 1406, c. 38r.); originariamente soprannome corrispondente all'aggettivo tergestino pičul, 'piccolo' (DORIA, Elementi 382), cfr. mugl. 120 pičul, FAGGIN 968 pičul.

Piçol pas, Piçolpass. Matia Piçol pas (R 1310 e Q 1318); Mocor Piçol paro (sic; Q 1316); Pascholus Piçolpass (C 1358); Servulus Parvipassi (C 1396); altre attestazioni: Dominicus Piçolpas (1354 BM). Soprannome composto con l'aggettivo tergestino pičul (si veda Piçol, Piçul) e il sostantivo *pas 'passo', cfr. mugl. 115 pas, FAGGIN 923 pas. Parvipassi è calco dotto latino.

Pleto, Pleti. Petrus Pleto/Pleti (R [1309-1310]); altre attestazioni: domus heredum Pleti (1325 MARSICH). Coincide con un aggettivo tergestino *plet 'gobbo', cfr. FAGGIN $9971^{\circ}$ plet 'curvo, gobbo'.

Plumaç. Çustol/Iustus Plumaç (R [1307-1310] e Q 1316-1317); Al(l)iandus Plumaç (R [1309]-1310); Ticolus Plumaç (R 1310, c. 2v.); altre attestazioni: Domina Andriota uxor Almerici Plumacii (1284- 1305 SSMM), ab Aymerico Plumacio (1308 SSMM, 673, c. 11v.). 
Il soprannome deriva da un sostantivo tergestino *pluma 'piuma', cfr. mugl. 123 plúma 'penna', FAGGIN 1003 plume '1. piuma; 2. pennuto; 3. mariolo, birba, briccone' e coincide con un sostantivo "plumač, cfr. FAGGIN 1003 plumačs 'piume, letto di piume'.

Qugumer. de morte Qugumer (Q 1318, c. 34v.); corrisponde a un sostantivo tergestino kukúmer 'cetriolo', cfr. mugl. 78 kukúmar 'cetriolo'.

Raiba. Vitalis Raiba/Rayba (R 1307-1309 e Q 1317-1319); Barcolus/Bartulus Raiba (R 1308 e Q 1319-1320); Andrea Rayba (R 1309); corrisponde a un sostantivo tergestino *raiba 'rabbia', cfr. FAGGIN 1058, 1061 raibe, raibie = rabie 'rabbia, ira, sdegno'.

Sbro(v)ada. Andreas Sbroada (C 1396, c. 29r.); Nicholaus Sbrovada (C 1403, c. 20r.); corrisponde a un sostantivo tergestino *sbroada o *sbrovada, cfr. mugl. 143 sbroáda 'piatto a base di verze inacidite' e FAGGIN 108, 1168 brovade, sbrovade 'rape inacetite'.

Scarabot. Tomaxina de Scarabot (D 1357, cc. 7r. e 14r.); corrisponde all'appellativo scorbuto o scarabàto 'malore che offende specialmente le viscere del corpo umano, che servono alla nutrizione' (BOERIO 649), ma si cfr. mugl. 153 skarakút 'scorbuto', quindi si pensa di poter risalire a un tergestino *scarabòt o *scarabùt.

Sonça de bec. Dionisius Sonça de bec ( $\mathrm{R} 1310$ e $\mathrm{Q}$ 1315-1320); Margareta/Margereta (uxor) de/quondam Sonça de bec(h) (R 1309-1310 e Q 1316). Soprannome composto con il sostantivo tergestino *son $3 a$, recepito dal triestino sonza 'sugna; fig. persona sudicia' (DORIA 1987, 649), la preposizione (triestina?) de e il sostantivo tergestino *bek (cfr. Bech); per il significato del quale si cita pure DORIA 1987, 65 2. beco 'becco (animale); fig. uomo cornuto'. Ma qui converrà risolvere il singolare soprannome col significato di 'uomo sudicio e puzzolente'.

Tric(h)a. Presbiter Tric(h)a (R [1309] e Q [1315]); Volricus/Tric(h)a (R 1309 e Q [1315] - 1317). Forse collegabile con il friulano triche 'briga, bega, lite, contesa, dissidio, contrasto' (FAGGIN 1518).

Çus. Stephanus/Steipano Çus (R 1310 e Q 1315). Coincide con lo zoonimo tergestino čus, zus 'assiolo; fig. babbeo' (DORIA, Elementi 363) e con il mugl. 165 sus 'gufo; allocco, persona goffa e balorda'. 


\section{FONTI}

Per i sette codici dell'Archivio Capitolare di S. Giusto in Trieste si useranno in seguito le segg. abbreviazioni:

$\mathbf{R}=$ Redditus et proventus proventus beneficiorum Canonicalium Ven. Capituli Tergestini de Anno 1310, codice di entrate e uscite [1307]-1310.

$\mathrm{Q}=$ Quaternus decimarum Ven. Capituli S. Iusti Mart. de Tergesto de Anno 1317, codice di entrate e uscite [1315]-1320.

$\mathrm{RP}=$ Redditus et proventus Canonicorum, et Capituli Ecclesice Targestince de 1377 et sparsim de pluribus annis sub eodem millesimo, codice di entrate e uscite del XIV secolo con date solo parzialmente accertabili presumibilmente dal 1338 al 1383.

$C=$ Receptus cere I (1357-1377), II (1389-1399), III (1400-1406; codici di entrate per diritti di stola nera.

$\mathrm{D}=$ Quaternus tam decimarum quam quartesiorum 1357, codice di entrate per il solo 1357 vergato dal canonico canipario Dominicus de Mianis.

Si citeranno inoltre altre fonti, nelle quali sono attestate le forme nominali interessate:

BLOISE = Delia Bloise, Testamenti trecenteschi delle XIII casate, in Archeografo triestino LXXXIX, 1980, pagg. 5-74.

BM = Lecia Persi Cocevar, Iacobus Gremon. Quaternus de defensionibus (1354), e I registri dei notai triestini dei Malefici Facina da Canciano a Iacobus Gremon (1352 e 1354), in Archeografo Triestino XCI (1982), 47-141 e 143-218.

HORTIS = Attilio Hortis, I Summaquesi, Guido de Guidis e Trieste (1277-1345), in Archeografo Triestino, Trieste 1882, pp. 364-404.

MAGISTRATURE = Delia Bloise, Giorgio Brischi, Annamaria Conti, Lucia Pillon, Michele Zacchigna, Le magistrature cittadine di Trieste nel secolo $X I V$, Guida e inventario delle fonti (con Indice dei nomi di persona a cura di Paolo Cammarosano), Trieste 1982.

MARSICH $=$ Don Angelo Marsich, Regesto delle pergamene conservate nell'Archivio del reverendissimo Capitolo della Cattedrale di Trieste, in Archeografo triestino, 1877-1885.

PARENTIN = Luigi Parentin, Regesto di 85 documenti inediti dell'Archivio Capitolare di Trieste, in Archeografo Triestino, 1965-1966, pp. 147-189.

PATRIZI = P(ietro) Kandler, Storia del Consiglio dei Patrizi di Trieste, Trieste 1858, ristampa fotomeccanica, Bologna 1971.

PILLON = Lucia Pillon, La fondazione della 'cella' di Trieste dalle origini alla metà del Quattrocento, tesi di laurea in storia medioevale, Università di Trieste, Anno accademico 1977-1978.

SSMM = Archivio del monastero benedettino dei SS. Martiri in Trieste, presso l'Archivio di Stato di Trieste, segn. C.R.S. Intendenza Commerciale per il Litorale in Trieste 1748 - 1776; segue il numero della busta e, dopo la barra, il numero del documento contenuto nella busta; solo per la busta 673 , contenente 
un catapan con documenti pergamenacei e cartacei del XIV secolo, dopo la barra segue la posizione della citazione sulla c(arta), suo numero, r(ecto) o v(erso).

Per i raffronti linguistici si citano pure:

BOERIO = Giuseppe Boerio, Dizionario del dialetto veneziano, Venezia 1856, ristampa anastatica, Firenze 1983.

DORIA 1987 = Mario Doria, Grande dizionario del dialetto triestino, Trieste 1987.

\section{Povzetek}

\section{TERGESTINSKA ANTROPONIMIKA V 14. STOLETJU}

Med številnimi imenskimi oblikami - priimki in vzdevki - v sedmih kodeksih prihodkov in razhodkov, ki jih hrani arhiv Stolnega kapitlja Sv. Justa $v$ Trstu in pokrivajo stoletje 1307-1406, so nekatere izražene $v$ tergestinščini. Tu objavljamo repertorij teh imenskih oblik in jih primerjamo $z$ ustreznimi besedami v dveh repertorijih tergestinske leksike Maria Doria, v muglizanskem narečnem besednjaku Diomira Zudinija in Pierpaola Dorsija ter $v$ dveh velikih furlanskih slovarjih, Pironovem in Fagginovem. Ker čaka Doriev tergestinski besednjak že več let na objavo, ne moremo ceniti količinskega in kakovostnega prispevka tega repertorija za poznavanje tergestinskega leksikalnega zaklada. 